\title{
GESTÃO DO TRABALHO EM SAÚDE: SENTIDOS E USOS DA EXPRESSÃO NO CONTEXTO HISTÓRICO BRASILEIRO
}

\author{
WORK MANAGEMENT IN HEALTHCARE: MEANING AND USE OF \\ EXPRESSION IN THE BRAZILIAN HISTORICAL CONTEXT
}

GESTIÓN DEL TRABAJO EN SALUD: SENTIDOS Y USO DE LA

EXPRESIÓN EN EL CONTEXTO HISTÓRICO BRASILEÑO

\author{
Dirce Laplaca Viana ${ }^{1}$ \\ Cleide Lavieri Martins ${ }^{2}$ \\ Paulo Frazão ${ }^{3}$
}

Resumo No Brasil, a expressão 'gestão do trabalho' passou a ser utilizada após a $12^{\text {a }}$ Conferência Nacional de Saúde em substituição a 'recursos humanos'. O objetivo foi analisar, com base no contexto histórico brasileiro, os sentidos atribuídos à expressão 'gestão do trabalho em saúde' na literatura científica brasileira, por meio de scoping review na base Literatura Latino-Americana e do Caribe em Ciências da Saúde (Lilacs), entre 1990 e 2010. De 436 estudos identificados, 34 foram incluídos. Houve crescimento absoluto das publicações após a $3^{a}$ Conferência Nacional de Gestão do Trabalho e Educação em Saúde, 2006. O conteúdo foi sintetizado em cinco unidades temáticas: conceitos de gestão do trabalho em saúde, formulação de políticas, participação dos trabalhadores nos processos de trabalho, precarização do trabalho e novos desafios. Observou-se consenso sobre a complexidade da expressão e os aspectos distintos foram explorados dependendo das características políticas, sociais e econômicas no momento da construção do pensamento. As discussões teóricas deveriam estimular mudanças nas práticas de gestão e cultura dos modelos de produção, porém as mudanças são escassas, restando um questionamento não desvelado: gestão do trabalho em saúde, uma mudança terminológica ou de paradigma? Palavras-chave administração de recursos humanos; administração de recursos humanos em saúde; gestão do trabalho em saúde; scoping review.
Abstract The expression 'work management in healthcare' began to be used in Brazil after the $12^{\mathrm{a}}$ National Conference of Health, replacing 'human resource'. The aim was analyze the meanings attributed to the use of the term 'work management in healthcare' based on the Brazilian historical context. This research developed a scoping review in the Lilacs database, between 1990 and 2010. Among 436 eligible studies, 34 were included. These publications presented numerical growth especially after the $3^{\text {rd }}$ National Conference of Work Management and Education in Healthcare at 2006. The content was synthetized in five main topics: conceptualizing of work management in healthcare, policy formulation, and participation of workers in the work process, labor precarization and new challenges. Despite the consensus about the complexity of the expression, distinct aspects are explored by the authors depending of political, social and economic contexts during the construction of thought. The theoretical discussions should support changes on practices of management and on culture of production modeling, but practical changes are scarce, generating a question that was not completely answered: does work management in healthcare represent a simple terminology exchange or paradigm shift?

Keywords personal management; health personal management; work management in health; scoping review. 


\section{Introdução}

O trabalho na área da saúde vem sofrendo várias transformações influenciadas pelas mudanças econômicas no sistema produtivo em geral e pelas reformas desse setor (Pires, 2000). No Brasil, apesar de temas relacionados ao trabalho em saúde já terem sido abordados nas conferências nacionais de saúde (CNS) de 1941 e 1950, somente receberam destaque em 1963, na $3^{a}$ CNS, e em 1986, na $8^{a}$ CNS, desde quando não saíram mais de pauta (Brasil, 1986a).

As discussões ocorridas na $8^{\mathrm{a}} \mathrm{CNS}$ influenciaram a realização da I Conferência Nacional de Recursos Humanos em Saúde (CNRHS), que destacou a relação entre valorização profissional e qualidade dos serviços, a regulação do mercado de trabalho, a implementação de políticas, a qualificação dos profissionais, a melhoria das condições de trabalho e as recompensas oferecidas por ele (Brasil, 1986b).

No relatório final da $12^{\mathrm{a}}$ CNS, publicado em 2004, foi possível constatar, pela primeira vez, o uso do termo 'gestão do trabalho' em detrimento do termo 'gestão de recursos humanos', com menção a uma possível crítica a esse último:

A gestão do trabalho e da educação na saúde é uma competência constitucional e legal dos gestores do SUS. Cabe ao Ministério da Saúde propor, acompanhar e executar as políticas de gestão do trabalho e de regulação profissional, de negociação do trabalho em saúde, junto com outros segmentos do governo e os trabalhadores, e o incentivo à estruturação de uma política de gestão do trabalho nas três esferas de governo, envolvendo os setores público e privado que compõem o SUS. (...) Saúde se faz com gente. Gente que cuida de gente, respeitando-se as diferenças de gênero, étnico-raciais e de orientação sexual. Por isso, os trabalhadores não podem ser vistos como mais um recurso na área da Saúde (Brasil, 2004a, p. 115).

No mesmo ano, foi criada a Secretaria de Gestão do Trabalho e da Educação na Saúde (SGTES), com o objetivo de implementar uma política de valorização do trabalho no Sistema Único de Saúde (SUS) e de seus trabalhadores, passando-se, dessa forma, a considerar a gestão do trabalho como uma questão estratégica (Pierantoni et al., 2008).

Em março de 2006, ocorreu o terceiro encontro nacional para discutir o tema 'recursos humanos em saúde', porém, houve mudança em seu nome, passando a ser denominado $3^{\text {a }}$ Conferência Nacional de Gestão do Trabalho e da Educação em Saúde (CNGTES). A crítica ao termo 'recursos humanos' foi reforçada nessa conferência, principalmente pelo então secretário da Secretaria de Gestão do Trabalho e da Educação em Saúde e coordenador da conferência, Francisco Eduardo Campos: “Os trabalhadores da saúde não são um insumo adicional que se agrega aos recursos financeiros, tecnológicos e 
de infraestrutura para produzir serviços: são os próprios serviços de saúde" (Dominguez, 2006, p. 8). Como resultado, a expressão 'recursos humanos' foi substituída definitivamente no nome da conferência e em todos os seus documentos por 'gestão do trabalho e da educação'.

Para Moyses e Machado (2010), embora se reconheça o termo 'recursos humanos', como consagrado na literatura mundial, não fazendo referência exclusivamente à sua conceituação clássica, a abordagem de gestão do trabalho corresponde melhor à dinâmica presente nas relações de construção e consolidação do SUS. Entende-se que mudanças na expressão sugerem também alterações no conceito e nas práticas de gestão; sendo assim, cabe analisar se essa dinâmica é expressa em novas pesquisas e se há, de fato, mudança nas práticas de gestão do trabalho.

Nessa perspectiva, sintetizar o conhecimento sobre os conceitos subjacentes à expressão 'gestão do trabalho em saúde' e os sentidos que lhes são atribuídos na produção científica publicada em periódicos brasileiros é importante para identificar diferenças e similaridades relacionadas à sua utilização pelos pesquisadores.

Para responder tais indagações, desenvolveu-se este estudo com o objetivo de analisar, com base no contexto histórico brasileiro, os sentidos atribuídos ao uso da expressão 'gestão do trabalho em saúde' na literatura científica.

\section{Percurso metodológico}

Trata-se de uma síntese da literatura por meio de scoping review, que compreende um conjunto de técnicas com a finalidade de sumarizar o conhecimento sobre um dado tema de investigação. Esse método foi esboçado pelas pesquisadoras Hilary Arksey e Lisa O'Malley em uma publicação de 2005, sendo referência importante para outras pesquisas (Arskey e O'Malley, 2005).

Esse método, ${ }^{4}$ assim como a maioria das revisões sistemáticas, é estruturado em cinco etapas: identificar a questão da pesquisa; identificar os estudos relevantes; selecionar os estudos; mapear os dados e confrontar, resumir e relatar os resultados, porém, com técnicas específicas para cada etapa (Viana, 2013).

Foram elaboradas duas questões para desenvolvimento desta pesquisa: $\mathrm{O}$ que se tem produzido no Brasil a respeito da gestão do trabalho em saúde? Quais os sentidos atribuídos ao uso dessa expressão na produção científica brasileira?

Para identificar os estudos relevantes foram utilizados os descritores 'gestão em saúde', 'administração em saúde' e 'trabalho', aplicados em busca na base de dados da Literatura Latino-americana e do Caribe em Ciências da Saúde (Lilacs), utilizando-se a seguinte composição: (gestão OR administração) AND (trabalho AND (saúde OR (serviços AND saúde))), filtrando por [palavras do título] OR [palavras do resumo] AND NOT teses [tipo de literatura]. O descritor 'gestão do trabalho' não foi localizado na taxonomia DeCS (Descritores em 
Ciências da Saúde). Foi considerado um período de 20 anos, escolhido com base no primeiro registro do uso da expressão (1991), por isso, a análise foi realizada entre 1990 e 2010.

Em busca eletrônica realizada em 5 de abril de 2011, foram encontradas 539 publicações. Por considerar que as teses resultam em artigos e que sua inclusão poderia causar duplicidade nos resultados, elas foram excluídas. Também foram excluídos livros, capítulos de livros e documentos governamentais porque poderiam não estar indexados em sua totalidade. Isso ocorre porque os livros e documentos são catalogados enquanto outros itens são indexados. As duas práticas têm como objetivo identificar o item e fornecer acesso a ele por meio de várias bases de dados, porém, na catalogação, o conteúdo é tratado no todo e os assuntos são fornecidos em uma escala limitada, enquanto na indexação a tendência é o detalhamento, em que há maior generosidade no fornecimento de termos para o acesso por assunto (Rubi, 2008). Fujita et al. (2009) observam que o usuário tem dificuldade de localizar livros por uma falha na catalogação. Por exemplo, livros com assuntos semelhantes não são catalogados pelo mesmo termo, prejudicando sua localização e a visibilidade de autores da mesma área de pesquisa. Sendo assim, foram excluídas da análise 103 publicações, muitas das quais foram consideradas na discussão dos resultados.

A seleção dos estudos ocorreu a partir da leitura dos títulos e resumos, e concomitante elaboração de critérios específicos de inclusão e exclusão, embora o scoping review permita estabelecer esses critérios posteriormente. Com base em 65 estudos potencialmente ligados ao tema, uma proposta de padronização de critérios foi elaborada por um dos autores. Em seguida, o autor principal e um dos coautores testaram essa proposta em uma amostra aleatória de 136 estudos. Para verificar o nível de concordância entre os dois examinadores, foram realizados testes de sensibilidade, especificidade e estatística kappa, obtendo-se respectivamente, os valores de $74 \%, 86,4 \%$ e 0,59 indicativos de boa concordância. Após a realização de pequenos ajustes, os critérios de inclusão e exclusão foram aprovados (Quadro 1).

\section{Quadro 1}

Critérios de inclusão e exclusão para seleção dos estudos sobre Gestão do Trabalho na Saúde. Brasil, 2011.

\begin{tabular}{|cr|}
\hline Critérios de inclusão & Critérios de exclusão \\
Abordar mercado de trabalho ou força de trabalho. & Não abordar gestão do trabalho. \\
Abordar processo de trabalho ou organização do trabalho. & Abordar cuidado assistencial ou saúde do trabalhador. \\
Enfocar estratégias de gestão relacionadas ao trabalho. & Abordar temas relacionados à formação \\
& profissional, epidemiologia ou vigilância em saúde.
\end{tabular}

Fonte: Os autores. 
Em seguida, os três autores aplicaram os critérios aos resumos dos 436 estudos e os classificaram como 'relacionado ao tema' e 'não relacionado ao tema'. Houve concordância em 322 artigos como 'não relacionados ao tema' e 40 artigos como 'relacionados ao tema'. Os estudos restantes (74) apresentaram resultados discordantes. Para estes, foi aplicada a técnica de consenso com classificação de 34 estudos como 'relacionados ao tema' e 40 como 'não relacionados ao tema'. Dessa forma, foram selecionados 74 artigos para leitura na íntegra.

O mapeamento dos dados incluiu o preenchimento de formulário contendo informações como: autoria, instituição do autor, título do estudo, periódico, ano de publicação, descritores/palavras-chave, abordagem metodológica, tipo de pesquisa, população estudada, período do estudo, referencial teórico, resultados obtidos e resultados importantes.

A Figura 1 apresenta as etapas do scoping review para seleção dos estudos.

Figura 1

Etapas do scoping review para a seleção dos estudos sobre Gestão do Trabalho em Saúde. Brasil, 2016.

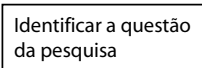

da pesquisa

Identificar estudos relevantes

Estabelecer critérios iniciais de exclusão

Elaborar e aplicar critérios de exclusão e

inclusão e selecionar

estudos

Mapear os dados

Confrontar, resumire

relatar os resultados
$\mathrm{O}$ que se tem produzido no Brasil a respeito

de gestão do trabalho em saúde?

Como essa expressão vem sendo utilizada

na produção científica brasileira?

Identificados 539 estudos relevantes sobre Gestão do Trabalho na Saúde

Excluídos 103 teses, livros, capítulos e documentos ministeriais

436 estudos para leitura do título e resumo

74 estudos para mapeamento

34 estudos para relatar os resultados

Resultados

Fonte: os autores

\section{Resultados}

Após a leitura dos 74 artigos, observou-se que 34 respondiam às questões norteadoras e, assim, foram considerados para confronto, relato e análise dos resultados (Quadro 2). 
Quadro 2

Artigos selecionados para o desenvolvimento do estudo sobre Gestão do Trabalho na Saúde. Brasil, 2016.

\begin{tabular}{|c|c|c|c|c|}
\hline & Autor(es) & Título & Periódico & Ano \\
\hline 1. & Barbosa, N. B. & $\begin{array}{l}\text { Regulação do trabalho no contexto das novas relações } \\
\text { público versus privado na saúde. }\end{array}$ & $\begin{array}{c}\text { Ciência \& Saúde } \\
\text { Coletiva }\end{array}$ & 2010 \\
\hline 2. & $\begin{array}{l}\text { Benito, G. A. V.; } \\
\text { Licheski, A.P. }\end{array}$ & $\begin{array}{l}\text { Sistemas de informação apoiando a gestão do trabalho } \\
\text { em saúde. }\end{array}$ & $\begin{array}{c}\text { Revista Brasileira } \\
\text { de Enfermagem }\end{array}$ & 2009 \\
\hline 3. & Campos, G. W. de S. & $\begin{array}{l}\text { Cogestão e neoartesanato: elementos conceituais } \\
\text { para repensar o trabalho em saúde combinando } \\
\text { responsabilidade e autonomia. }\end{array}$ & $\begin{array}{c}\text { Ciência \& Saúde } \\
\text { Coletiva }\end{array}$ & 2010 \\
\hline 4. & $\begin{array}{l}\text { Campos, G. W. de S.; } \\
\text { Domitti, A. C. }\end{array}$ & $\begin{array}{l}\text { Apoio matricial e equipe de referência: uma metodologia } \\
\text { para gestão do trabalho interdisciplinar em saúde. }\end{array}$ & $\begin{array}{c}\text { Cadernos de Saúde } \\
\text { Pública }\end{array}$ & 2007 \\
\hline 5. & $\begin{array}{l}\text { Duarte, I. G.; } \\
\text { Botazzo, C. }\end{array}$ & $\begin{array}{l}\text { Gestão de pessoas nas Organizações Sociais de Saúde: } \\
\text { algumas observações. }\end{array}$ & $\begin{array}{l}\text { Revista de } \\
\text { Administração em } \\
\text { Saúde }\end{array}$ & 2009 \\
\hline 6. & $\begin{array}{l}\text { Fernandes, M. C. } \\
\text { et al. }\end{array}$ & $\begin{array}{l}\text { Análise da atuação do enfermeiro na gerência de } \\
\text { unidades básicas de saúde. }\end{array}$ & $\begin{array}{l}\text { Revista Brasileira } \\
\text { de Enfermagem }\end{array}$ & 2010 \\
\hline 7. & $\begin{array}{l}\text { Garibotti, V.; } \\
\text { Hennington, E. A.; } \\
\text { Selli, L. }\end{array}$ & $\begin{array}{l}\text { A contribuição dos trabalhadores na consolidação dos } \\
\text { serviços municipais de vigilância sanitária. }\end{array}$ & $\begin{array}{c}\text { Cadernos de Saúde } \\
\text { Pública }\end{array}$ & 2006. \\
\hline 8. & Hennington, E. A. & $\begin{array}{l}\text { Gestão dos processos de trabalho e humanização em } \\
\text { saúde: reflexões a partir da ergologia. }\end{array}$ & $\begin{array}{l}\text { Revista de Saúde } \\
\text { Pública }\end{array}$ & 2008 \\
\hline 9. & Jorge, M. S. B. et al. & $\begin{array}{l}\text { Gestão de recursos humanos nos centros de atenção } \\
\text { psicossocial no contexto da política de desprecarização } \\
\text { do trabalho no Sistema Único de Saúde. }\end{array}$ & $\begin{array}{l}\text { Texto \& Contexto } \\
\text { Enfermagem }\end{array}$ & 2007 \\
\hline 10. & Junqueira, T. S. et al. & $\begin{array}{l}\text { As relações laborais no âmbito da municipalização da } \\
\text { gestão em saúde e os dilemas de relação expansão/ } \\
\text { precarização do trabalho no contexto do SUS. }\end{array}$ & $\begin{array}{c}\text { Cadernos de Saúde } \\
\text { Pública }\end{array}$ & 2010 \\
\hline 11. & Junqueira, T. S. et al. & $\begin{array}{l}\text { Saúde, democracia e organização do trabalho no contexto } \\
\text { do Programa de Saúde da Família: desafios estratégicos. }\end{array}$ & $\begin{array}{l}\text { Revista Brasileira de } \\
\text { Educação Médica }\end{array}$ & 2009 \\
\hline 12. & Laca: & $\begin{array}{l}\text { Qualidade de vida, gestão do trabalho e plano de } \\
\text { carreira como tecnologista em saúde na atenção básica } \\
\text { do Sistema Único de Saúde em São Paulo, Brasil. }\end{array}$ & $\begin{array}{c}\text { Cadernos de Saúde } \\
\text { Pública }\end{array}$ & 2010 \\
\hline 13. & 1. $\mathrm{H}$. & as. & $\begin{array}{l}\text { Administração } \\
\text { Pública }\end{array}$ & 2000 \\
\hline 14. & $\begin{array}{l}\text { Machado, M. H.; Paula } \\
\text { A. E.; Aguiar Filho, W. }\end{array}$ & $\begin{array}{l}\text { O trabalho em saúde no Mercosul: uma abordagem } \\
\text { brasileira sobre a questão. }\end{array}$ & $\begin{array}{c}\text { Cadernos de Saúde } \\
\text { Pública }\end{array}$ & 2007 \\
\hline 15. & Matos E.; PireS, D. & $\begin{array}{l}\text { Teorias administrativas e organização do trabalho: de Taylor } \\
\text { aos dias atuais, influências no setor saúde e na enfermagem. }\end{array}$ & $\begin{array}{l}\text { Texto \& Contexto } \\
\text { Enfermagem }\end{array}$ & 2006 \\
\hline 16. & $\begin{array}{l}\text { Medeiros, A. C. de } \\
\text { et al. }\end{array}$ & $\begin{array}{c}\text { Gestão participativa na educação permanente em } \\
\text { saúde: olhar das enfermeiras. }\end{array}$ & $\begin{array}{l}\text { Revista Brasileira } \\
\text { de Enfermagem }\end{array}$ & 2010 \\
\hline 17. & $\begin{array}{l}\text { Mello, M. L. B. C. de; } \\
\text { AMÂNCIO FILHO, A. }\end{array}$ & $\begin{array}{l}\text { A gestão de recursos humanos em uma instituição } \\
\text { pública brasileira de ciência e tecnologia em saúde: o } \\
\text { caso Fiocruz. }\end{array}$ & $\begin{array}{l}\text { Revista de } \\
\text { Administração } \\
\text { Pública }\end{array}$ & 2010 \\
\hline 18. & $\begin{array}{l}\text { Mendonça, M. H. } \\
\text { M. et al. }\end{array}$ & $\begin{array}{l}\text { Desafios para a gestão do trabalho a partir de } \\
\text { experiências exitosas de expansão da Estratégia de } \\
\text { Saúde da Família. }\end{array}$ & $\begin{array}{l}\text { Ciência \& Saúde } \\
\text { Coletiva }\end{array}$ & 2010 \\
\hline
\end{tabular}


Continuação - Quadro 2

\begin{tabular}{|c|c|c|c|c|}
\hline 19. & $\begin{array}{l}\text { Moyses, N. M. N.; } \\
\text { Machado, M. H. }\end{array}$ & $\begin{array}{l}\text { Políticas de gestão do trabalho no SUS: o desafio sempre } \\
\qquad \text { presente. }\end{array}$ & $\begin{array}{l}\text { Divulgação em } \\
\text { Saúde para Debate }\end{array}$ & 2010 \\
\hline 20. & Pereira, L. D. & A gestão da força de trabalho em saúde na década de 90. & $\begin{array}{l}\text { Physis: Revista de } \\
\text { Saúde Coletiva }\end{array}$ & 2004 \\
\hline 21. & Pierantoni, C. R. & $\begin{array}{l}\text { As reformas do Estado, da saúde e recursos humanos: } \\
\text { limites e possibilidades. }\end{array}$ & $\begin{array}{c}\text { Ciência \& Saúde } \\
\text { Coletiva }\end{array}$ & 2001 \\
\hline 22. & Pierantoni, C. R. et al. & $\begin{array}{c}\text { Gestão do trabalho e da educação em saúde: recursos } \\
\text { humanos em duas décadas do SUS. }\end{array}$ & $\begin{array}{l}\text { Physis: Revista de } \\
\text { Saúde Coletiva }\end{array}$ & 2008 \\
\hline 23. & $\begin{array}{l}\text { Pimenta, A. L.; } \\
\text { Livorato, F. }\end{array}$ & $\begin{array}{l}\text { Educação permanente: ferramenta para qualificar os } \\
\text { sistemas municipais de saúde. }\end{array}$ & $\begin{array}{l}\text { Divulgação em } \\
\text { Saúde para Debate }\end{array}$ & 2009 \\
\hline 24. & Pires, D. & $\begin{array}{c}\text { Reestruturação produtiva e consequências para o } \\
\text { trabalho em saúde. }\end{array}$ & $\begin{array}{l}\text { Revista Brasileira } \\
\text { de Enfermagem }\end{array}$ & 2000 \\
\hline 25. & $\begin{array}{l}\text { Pires-Alves F.; Paiva, } \\
\text { C. H. A.; Hochman, G. }\end{array}$ & $\begin{array}{l}\text { História, saúde e seus trabalhadores: da agenda } \\
\text { internacional às políticas brasileiras. }\end{array}$ & $\begin{array}{c}\text { Ciência \& Saúde } \\
\text { Coletiva }\end{array}$ & 2008 \\
\hline 26. & Ramos, A. S. et al. & $\begin{array}{l}\text { Política de gestão do trabalho e educação permanente } \\
\text { na Bahia: "o SUS é uma escola”. }\end{array}$ & $\begin{array}{l}\text { Revista Baiana de } \\
\text { Saúde Pública }\end{array}$ & 2009 \\
\hline 27. & Santana, J. P. & Gestão do trabalho no serviço público de saúde. & Saúde em Debate & 1992 \\
\hline 28. & $\begin{array}{l}\text { Scalco, S. V.; Lacerda, } \\
\text { J.T. de; Calvo, M. C. M. }\end{array}$ & $\begin{array}{c}\text { Modelo para avaliação da gestão de recursos humanos } \\
\text { em saúde. }\end{array}$ & $\begin{array}{l}\text { Cadernos de Saúde } \\
\text { Pública }\end{array}$ & 2010 \\
\hline 29. & $\begin{array}{c}\text { Scherer, M. D. A.; } \\
\text { Pires, D.; Schwartz, Y. }\end{array}$ & Trabalho coletivo: um desafio para a gestão em saúde. & $\begin{array}{l}\text { Revista de Saúde } \\
\text { Pública }\end{array}$ & 2009 \\
\hline 30. & Silveira, D. S. et al. & $\begin{array}{l}\text { Gestão do trabalho, da educação, da informação e } \\
\text { comunicação na atenção básica à saúde de municípios } \\
\text { das regiões Sul e Nordeste do Brasil. }\end{array}$ & $\begin{array}{l}\text { Cadernos de Saúde } \\
\text { Pública }\end{array}$ & 2010 \\
\hline 31. & Sodré, F. & $\begin{array}{l}\text { Serviço social e o campo da saúde: para além de } \\
\text { plantões e encaminhamentos. }\end{array}$ & $\begin{array}{l}\text { Serviço Social \& } \\
\text { Sociedade }\end{array}$ & 2010 \\
\hline 32. & Timbó, D. B. P. et al. & $\begin{array}{l}\text { As competências para o gerenciamento eficaz: o caso da } \\
\text { Rede de Serviços de Saúde São Gerardo. }\end{array}$ & $\begin{array}{l}\text { Revista de } \\
\text { Administração em } \\
\text { Saúde }\end{array}$ & 2009 \\
\hline 33. & $\begin{array}{l}\text { Viegas, S. M. da F. } \\
\text { et al. }\end{array}$ & $\begin{array}{l}\text { O cotidiano da assistência ao cidadão na rede de saúde } \\
\text { de Belo Horizonte. }\end{array}$ & $\begin{array}{l}\text { Physis: Revista de } \\
\text { Saúde Coletiva }\end{array}$ & 2010 \\
\hline 34. & Vieira, M. & $\begin{array}{l}\text { Trabalho, qualificação e a construção social de identidades } \\
\text { profissionais nas organizações públicas de saúde. }\end{array}$ & $\begin{array}{l}\text { Trabalho, } \\
\text { Educação e Saúde }\end{array}$ & 2007 \\
\hline
\end{tabular}

Fonte: Os autores.

A apresentação dos resultados foi dividida em duas partes. Na primeira descrevem-se as características da produção científica, e na segunda apresentam-se os sentidos atribuídos ao uso da expressão 'gestão do trabalho em saúde'. Essa análise gerou cinco unidades temáticas a partir do exame do conteúdo dos textos e do estabelecimento de relações de independência e pertinência temática entre eles: conceito de 'gestão do trabalho em saúde', formulação de políticas, participação dos trabalhadores nos processos de trabalho (desalienação e autonomia), precarização do trabalho e novos desafios. 
Conforme se pode observar no Quadro 2, os autores que mais publicaram sobre gestão do trabalho em saúde no período estudado foram Denise Elvira Pires de Pires, professora titular da Universidade de Santa Catarina, e Maria Helena Machado, pesquisadora titular da Escola Nacional de Saúde Pública Sergio Arouca da Fundação Oswaldo Cruz (Fiocruz), com três publicações cada uma.

O estado com mais produções sobre o tema foi o Rio de Janeiro, representado por 14 publicações, sendo nove vinculadas à Fiocruz, seguido pelos estados do Rio Grande do Sul (nove), São Paulo (sete), Ceará (sete), Santa Catarina (seis) e Minas Gerais (cinco). Constatou-se que 31 artigos estavam vinculados a instituições de ensino e seus programas de pós-graduação. De fato, mais da metade dos cursos de doutorado, mestrado profissional e mestrado acadêmico estão localizados na Região Sudeste, seguida numericamente pelas regiões Sul, Nordeste, Centro-Oeste e Norte (Brasil, 2010).

O periódico Cadernos de Saúde Pública apresentou o maior número de publicações (sete/ 20,6\%), seguido pela revista Ciência \& Saúde Coletiva, vinculada à Associação Brasileira de Saúde Coletiva (cinco/ 14,7\%) e pela Revista Brasileira de Enfermagem, vinculada à Associação Brasileira de Enfermagem (quatro/ 11,7\%), ou seja, 47\% dos artigos foram publicados em três periódicos; os demais estavam distribuídos em 12 periódicos nacionais diferentes.

Com relação ao período considerado para busca, o Gráfico 1 mostra que as publicações sobre o tema apresentaram crescimento absoluto a partir de 2006, o que pode ter relação com o advento da $3^{\mathrm{a}} \mathrm{CNGTES}$, e se mantiveram em alta desde então.

\section{Gráfico 1}

Distribuição numérica da produção sobre gestão do trabalho na saúde publicada no Brasil na base de dados Lilacs por ano, Brasil, 2016.

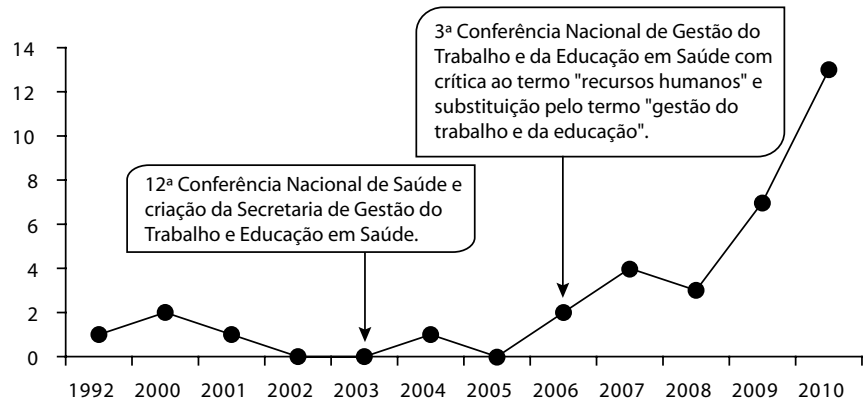

Fonte: Os autores

Dos 34 artigos analisados, 27 utilizaram palavras-chave, seis utilizaram descritores e um não registrou qualquer termo. A utilização de palavras-chave ou descritores depende das normas para publicação do periódico. Descritores 
são termos definidos por especialista e criteriosamente catalogados, com suas descrições, origens, significados e relações com outros descritores, em bases de dados específicas, enquanto as palavras-chave são termos escolhidos livremente pelo autor para definir assuntos relacionados a uma publicação específica. Os descritores mais utilizados foram 'Gestão em saúde' (quatro vezes) e 'Recursos humanos em saúde' (duas vezes). Os demais textos apresentaram variação de descritores, em um total de 21 diferentes. As palavras-chave mais utilizadas também foram 'Gestão em saúde' e 'Recursos humanos em saúde', apontadas em sete artigos cada uma. Foram identificadas 68 palavras-chave diferentes nos textos estudados.

Dos artigos incluídos, 29 utilizaram a abordagem qualitativa, dois a quantitativa e três a quanti-qualitativa. Dois artigos eram ensaios, 13 utilizaram a pesquisa bibliográfica como tipo de investigação, 11 empregaram a pesquisa de campo, sete se apoiaram no estudo de caso e um foi pesquisa de levantamento (survey).

Os artigos apresentaram 705 referências diferentes, sendo 155 documentos oficiais (publicações da Organização Mundial da Saúde (OMS), Organização Pan-Americana de Saúde (Opas), dos ministérios, secretarias estaduais e municipais, leis e portarias, entre outros) com destaque para 81 publicações do MS (52\%), incluindo publicações da SGTES, Constituição da República Federativa do Brasil de 1.988 e os Princípios e Diretrizes para a Gestão do Trabalho no SUS (Norma Operacional Básica de Recursos Humanos - NOB/RH-SUS).

Os autores mais citados foram: Roberto Passos Nogueira, com 17 publicações diferentes; Maria Helena Machado, com 16 publicações; Gastão Wagner de Souza Campos, com 12 publicações.

Algumas referências presentes nos artigos eram reincidentes. Esse cenário pode estar relacionado ao fato de que os autores mais citados também assinaram publicações vinculadas ao Ministério da Saúde (MS) ou a Opas, órgãos que, por suas características, têm forte influência na definição das políticas de saúde. Percebeu-se, então, que o tema é predominantemente explorado por um grupo específico de especialistas.

\section{Conceito de gestão do trabalho em saúde}

Das 34 publicações, dez apresentaram o conceito de gestão do trabalho em saúde como estabelecido ou como conceito ainda em construção, porém, antes de iniciar a discussão a respeito do conceito de gestão do trabalho em saúde, é necessário conhecer os aspectos que envolvem a concepção de um conceito.

Com base nos estudos de Reinhart Koselleck, pesquisador alemão que se dedicou à reflexão teórico-metodológica a respeito da história dos conceitos (Begriffsgeschichte), um conceito está diretamente relacionado àquilo que se quer compreender, ou seja, não apenas como um fenômeno linguístico, mas 
também considerando fatores como, por exemplo, conflitos políticos e sociais existentes no momento em que esse conceito foi pensado, falado ou expressado. Além disso, os conceitos podem ser alterados com o passar do tempo e influenciados pela experiência de quem o utilizou, recebeu e interpretou (Koselleck, 1992; Jasmin, 2005).

A expressão 'gestão do trabalho' foi utilizada pela primeira vez em uma publicação de Fischer (1991), na área da administração de empresas. O texto enfoca o trabalho e sua gestão como fator estratégico para o processo de modernização das organizações complexas, destacando a necessidade de se valorizar o fator trabalho que, segundo a autora, constitui pedra de toque para repensar a política de gestão no Brasil, tanto em nível institucional quanto organizacional.

Ainda, segundo a autora, há necessidade de se identificar o complexo jogo de influências que ocorre tanto em nível institucional quanto organizacional a fim de compreender os padrões de gestão do trabalho e sua contribuição para o desenvolvimento econômico e social do país (Fischer, 1991, p.86).

Especificamente voltada à saúde, essa expressão foi utilizada pela primeira vez em um artigo de Santana (1992), que relaciona a deterioração da qualidade dos serviços de saúde a três aspectos distintos, porém interligados: redução do gasto e do investimento público, aplicação desordenada ou inadequada de novas tecnologias na área da saúde e deficiência de disponibilidade e uso de recursos humanos.

Os autores citados, apesar de inseridos em contextos diferentes, concordam com relação à necessidade de superar entraves e limitações presentes nos processos de gestão do trabalho, levando-se em consideração as peculiaridades de cada setor de atividade.

Uma publicação de 2004, feita pela SGTES, afirmou que a gestão do trabalho em saúde diz respeito a todos os aspectos relacionados à atividade laboral, aos atores envolvidos e suas relações, considerando, além da questão técnica, as mudanças que ocorrem a partir dessas relações, sejam nos processos de execução da atividade em si, nas ações dos envolvidos ou, principalmente, nas pessoas. Após essa publicação, outros especialistas passaram a empregar esse conceito como base para discussão sobre o tema (Brasil, 2004b; Benito e Licheski, 2009; Junqueira et al., 2009; Medeiros et al., 2010).

A gestão do trabalho em saúde pode ser considerada, então, como uma ação de âmbito macrossocial, sob a responsabilidade do Estado, e mesossocial, envolvendo sindicatos, associações, entidades e instituições, cujos efeitos incidem sobre os usuários, os trabalhadores, os gestores dos serviços e do sistema, ou seja, contemplando também a dimensão microssocial (Nogueira, 2002).

Ressalta-se, porém, que existe uma dependência da ação do Estado, que regula a ordem social e econômica e, tradicionalmente, intervém nas relações de trabalho e capital; e, como consequência, "as práticas de gestão tendem a reduzir-se à postura legislativa que define o valor do fator trabalho e o padrão 
das relações estabelecidas no âmbito organizacional, exclusivamente, em função da obrigatoriedade das normas jurídicas efetivas" (Fischer, 1991, p. 88).

Para Santana (1992), há que se ter uma visão ampla acerca do tema, considerando ao menos três dimensões: econômica, enquanto relação de troca entre trabalho e salário; político-ideológica, enquanto relação de poder e espaço de conflitos; e organizativa do processo produtivo, enquanto relações interpessoais decorrentes da divisão do trabalho. Além disso, é necessário considerar o contexto atual de um mundo organizado sob a hegemonia do modo capitalista de produção (Pires, 2000).

Outros aspectos conceituais da gestão do trabalho são abordados, incluindo a formulação de políticas, a participação dos trabalhadores, sua desalienação e autonomia e a precarização do trabalho.

Segundo Pires-Alves et al. (2008), o próprio uso da expressão 'trabalhadores da saúde', que se tornou hegemônico em relação a recursos humanos ou força de trabalho, é um fenômeno histórico a ser explicado.

De uma maneira geral, pode-se afirmar que existe consenso entre os autores de que gestão do trabalho em saúde diz respeito ao trabalhador e seu trabalho, incluindo a valorização do trabalho e do trabalhador por meio da regulação do trabalho com condições adequadas e sua desprecarização, além de envolver toda a vida funcional do trabalhador, desde o seu ingresso no mercado de trabalho até a garantia dos direitos trabalhistas, perpassando a capacitação, formação, participação nos processos de trabalho e nas formulações de políticas, contribuindo para sua desalienação.

Apesar dos pontos consensuais, alguns autores enfocam aspectos distintos relacionados à 'gestão do trabalho em saúde', reforçando a ideia da historicidade do conceito, sua estreita relação com valores políticos, teorias sociais, mudanças e inovações envolvendo as transformações estruturais do governo, da sociedade e da economia e as aplicações decorrentes no mundo do trabalho.

\section{Formulação de políticas em gestão do trabalho em saúde}

A formulação de políticas voltadas à gestão do trabalho em saúde fundamentou as discussões de nove publicações. De acordo com Saraiva (2006), a formulação de políticas mostra-se como forma moderna de lidar com incertezas decorrentes das rápidas mudanças do contexto, principalmente no mundo do trabalho.

Apesar de existirem inúmeras definições para o que seja política pública, Saraiva (2006) afirma que diz respeito ao fluxo de decisões formuladas com o intuito de manter o equilíbrio social ou iniciar desequilíbrios para que a realidade seja modificada.

Na saúde, nota-se preocupação com as políticas públicas voltadas ao trabalho, que figura desde documentos de 1963; porém, somente na $10^{\text {a }} \mathrm{CNS}$, 
ocorrida em 1996, se propôs que fosse desenvolvida uma Política de Recursos Humanos para a Saúde e uma NOB/RH com princípios que regulassem a ação e relação das esferas de governo no que diz respeito aos trabalhadores do SUS (Brasil, 1998).

Em novembro de 1998, foi publicada a primeira versão do documento "Princípios e Diretrizes para NOB/ RH - SUS", que viria a ser a Política de Recursos Humanos para a Saúde. A segunda versão desse documento foi publicada em 2000 e, alguns anos depois, por meio da resolução n. 330, de 4 de novembro de 2003, o Conselho Nacional de Saúde resolveu considerar esse documento como Política Nacional de Gestão do Trabalho e da Educação em Saúde no âmbito do SUS, publicada em 2005 como a terceira e última versão (Brasil, 2005), vigente ainda em 2016.

Em 2003, a criação da SGTES deu à gestão do trabalho um tratamento estratégico. Essa que passou a ser vista como política de Estado, tanto na dimensão técnica, quanto nas relações de trabalho, considerando as diferenças culturais e regionais e suas implicações como centrais na dinâmica do SUS (Machado et al., 2007; Ramos et al., 2009; Mendonça et al., 2010; Scalco; Lacerda e Calvo, 2010).

Como resultado de toda essa movimentação em prol da adoção de uma política de gestão do trabalho em saúde, muitos estados brasileiros iniciaram processos para o desenvolvimento de políticas locais, porém de forma tímida, e muito vulnerável aos interesses da sociedade e aos impostos pela política econômica (Jorge et al., 2007). De uma maneira geral, pode-se afirmar que a formulação de políticas voltadas à gestão do trabalho em saúde representou importante iniciativa que poderá inspirar modificações relacionadas tanto à desprecarização do trabalho, quanto à implementação de planos de cargos, carreiras e salários, com forte potencial de produção de impacto na prática profissional a depender dos desdobramentos e das experiências de mudança.

\section{Participação dos trabalhadores nos processos de trabalho}

Muitas publicações a respeito dos trabalhadores, principalmente da saúde, destacam a autonomia profissional como forma de impedir que permaneçam alienados dos processos de trabalho e afastados das decisões tomadas dentro das instituições. Dentre os artigos consultados, 17 abordam esse tema de maneira enfática e consideram a participação dos trabalhadores nos processos como única maneira de elevar a qualidade do trabalho em saúde.

A alienação é resultado da divisão de tarefas em partes fragmentadas do processo de trabalho, atrelada à fixação do profissional em uma determinada etapa deste processo e à perda de controle dos meios de produção, da visão do todo e de como o trabalho de cada um está inserido nesse todo (Matos e Pires, 2006; Viegas et al., 2010). 
A divisão do trabalho dificulta a integração do processo de atenção e cuidado às pessoas; além disso, impossibilita a integração dialógica entre distintas especialidades e profissões (Campos e Domitti, 2007).

Campos (2010) ressalta que as instituições contemporâneas estimulam a implementação de protocolos que servem de condutores de comportamentos. Essa racionalidade gerencial hegemônica busca, de maneira utópica,

reduzir o sujeito do trabalho a um objeto, a um recurso maleável conforme o planejamento e programas definidos pela direção ou por 'quem entende do assunto', em geral, especialistas que pensam e planejam afastados do espaço onde se realiza o trabalho (Campos, 2010, p. 2.338).

Porém, mesmo com protocolos e regras tão comuns à prática na área da saúde, não há possibilidade de padronização total dos procedimentos, visto que o cotidiano de trabalho exige que seus trabalhadores muitas vezes arbitrem sobre suas ações (Hennington, 2008).

Ademais, apesar de outras metodologias administrativas terem sido difundidas principalmente a partir da década de 1980, especialmente quanto à participação dos trabalhadores nos processos de tomada de decisões, as grandes instituições hospitalares realizam a gestão do trabalho com os mesmos métodos adotados em grandes fábricas, ou seja, atendimento em massa, cirurgias em massa, internações contabilizadas pelo seu gasto financeiro, leitos em série e atendimentos sequenciais sem tempo de parada (Machado, 2000; Sodré, 2010).

Em contraposição, pode-se afirmar que, na gestão do trabalho, o trabalhador deve ser entendido como um "sujeito e agente transformador de seu ambiente e não apenas um recurso humano realizador de tarefas previamente estabelecidas pelas esferas maiores" (Benito e Licheski, 2009, p.448).

Uma das estratégias que oferecem ferramentas para que os profissionais possam arbitrar e tomar a decisão mais adequada ao caso que se apresenta é a educação permanente em saúde que, de acordo com Medeiros et al. (2010), deve ser utilizada como estratégia de gestão do trabalho e da educação em saúde porque possibilita o desenvolvimento permanente dos trabalhadores.

Porém, a educação permanente exige ações para além da formação técnica, envolvendo a organização do trabalho, a interação entre as redes de gestão e de serviços de saúde e o controle social neste setor. Além disso, deve visualizar o ser humano, meio ambiente e integração entre os diversos saberes (Brasil, 2004b; Fernandes et al., 2010).

Para Pierantoni (2001), a autonomia profissional está relacionada tanto à autonomia técnica quanto à política e econômica, com modelos mais democráticos de relações interpessoais com a participação de todos, mesmo que estes assumam papéis decisórios distintos. 
Diante do exposto, há que se estimular perfis de gestão com a participação dos profissionais nos processos de trabalho junto aos gestores e representantes das instituições de saúde, visto que não é possível mudar tais características alienatórias sem que a cultura das organizações seja também modificada.

\section{Precarização do trabalho}

Temas relacionados à precarização do trabalho em saúde têm recebido cada vez mais destaque nas discussões entre gestores e atores envolvidos. Dos 34 artigos utilizados, nove discutem questões relacionadas à precarização do trabalho como, por exemplo, os prejuízos para o setor saúde e as principais formas de reverter esse problema.

No último século, foi possível perceber que a sociedade do trabalho sofreu mudanças importantes, porém, contraditórias. O mundo moderno evidenciou a valorização do trabalho intelectual em detrimento do trabalho manual, e o trabalhador passou a ser estimulado a desenvolver atividades mais intelectualizadas, complexas, autônomas e coletivas, ao mesmo tempo em que foi possível identificar o aumento estrutural do desemprego e do trabalho precário e informalizado (Mello e Amâncio Filho, 2010).

No Brasil, com a entrada em vigor da Constituição de 1988, ocorreu a descentralização do SUS, havendo a transferência da gestão do trabalho para os municípios. Isso, por um lado, provocou a expansão das contratações dos trabalhadores, mas, por outro, expôs os trabalhadores a diversas modalidades contratuais frágeis, inadequação quantitativa do quadro de pessoal, baixa qualificação profissional, alta rotatividade, absenteísmo e baixa motivação, entre outros problemas (Pierantoni et al., 2008; Duarte e Botazzo, 2009; Machado e Koster, 2011).

As variadas formas de contratação representam o processo mais concreto de precarização das relações de trabalho na saúde, que se tornam ainda mais evidentes quando ocorrem no mesmo ambiente de trabalho, podendo, inevitavelmente, gerar relações acirradas de competitividade e descontentamento entre os trabalhadores (Pereira, 2004; Silveira et al., 2010).

Uma pesquisa realizada por Junqueira et al. (2010) discutiu os dilemas da relação expansão/precarização do trabalho no contexto do SUS; mostrou a precarização nas áreas de gestão do trabalho relacionadas com o recrutamento e seleção de profissionais, formas de vinculação, rotatividade principalmente de médicos e enfermeiros; remuneração, carga horária de trabalho e política de capacitação.

O MS reconhece os prejuízos da precarização do trabalho quando afirma que

o trabalho precário em saúde tem sido identificado como um obstáculo para o desenvolvimento do sistema público de saúde. Essa questão compromete a relação dos trabalhadores com o sistema e prejudica a qualidade e a continuidade dos serviços essenciais prestados pelo SUS (Brasil, 2006, p.12). 
A partir desse cenário, surgiram iniciativas como a criação, em 2003, do Comitê Nacional Interinstitucional de Desprecarização do Trabalho no SUS e, no mesmo ano, durante a $12^{\text {a }}$ CNS, o MS também defendeu a implementação de uma Política de Desprecarização do Trabalho em Saúde, principalmente no que diz respeito aos vínculos trabalhistas, implementação do Plano de Carreira, Cargos e Salários do SUS, desenvolvimento de ações, mesas de negociação em processos decisórios, avaliação de desempenho e criação de instrumentos com o objetivo de humanizar as relações entre profissionais e usuários (Pereira, 2004; Lacaz et al., 2010).

Em 2006, por meio da SGTES, o MS criou o Programa Nacional de Desprecarização do Trabalho no SUS, conhecido como DesprecarizaSUS, com o objetivo de buscar soluções para as situações de precarização dos vínculos de trabalho nas três esferas do governo (Brasil, 2006; Junqueira et al., 2010).

Tais iniciativas têm chamado atenção dos gestores para a importância de desprecarizar o trabalho na saúde, principalmente no SUS, com o objetivo de oferecer melhores condições de trabalho aos clientes internos e, consequentemente, um serviço de qualidade aos clientes externos.

Porém, apesar dessas iniciativas, cabe salientar que, para que haja de fato desprecarização do trabalho, faz-se necessário rever aspectos relacionados às formas de contratação e vinculação do trabalhador; à remuneração e jornada de trabalho compatíveis com o mercado e com a manutenção da qualidade do serviço, incluindo política de educação permanente; à instituição de planos de carreira, cargos e salários ajustados às exigências do SUS; e ainda aos programas de avaliação de desempenho, dentre outros aspectos.

\section{Novos desafios}

Muitos estudos incluídos nesta pesquisa apontam a gestão do trabalho em saúde como uma das mais importantes, complexas e desafiadoras áreas que compõem uma organização, recebendo destaque em 18 artigos estudados.

As estruturas relacionadas ao mercado e às políticas de gestão do trabalho sofreram nítidas e importantes transformações nos últimos anos, mas, mesmo assim, não estão completamente resolvidas e ainda apresentam fragilidades, principalmente nos aspectos que envolvem as estratégias para responder às transformações que vêm ocorrendo no mundo contemporâneo e na sociedade, ao mesmo tempo em que o trabalhador vem sendo considerado fator importante para o sucesso organizacional. Além disso, ainda não existem soluções a respeito da melhor maneira de lidar com as especificidades do setor público de saúde (Pierantoni et al., 2008; Timbó et al., 2009).

Ainda que as mudanças no mundo corporativo afetem as relações produtivas nos diversos níveis da economia, destaca-se a importância de recuperar e ideia de trabalho como fonte de realização (Vieira, 2007). 
O aumento no número de pesquisas e estudos sobre gestão do trabalho, publicados a partir da década de 1990, foi motivado pelas mudanças ocorridas no mercado em geral. As discussões que receberam mais destaque envolveram a crescente informalidade das atividades profissionais, incluindo o trabalho no SUS, que também segue a tendência geral de desregulação das relações (Barbosa, 2010).

Com relação à gestão do trabalho em saúde, Scherer et al. (2009, p. 725) afirmam que os principais desafios dizem respeito "à relação entre sujeitos individuais e coletivos; à história das profissões de saúde e o seu exercício no cenário do trabalho coletivo institucionalizado e à complexidade do jogo político econômico que delimita o cenário das situações de trabalho".

A gestão do trabalho na saúde deve ser tratada como área estratégica e fundamental para o efetivo funcionamento das organizações no que diz respeito ao atendimento das demandas reais, com estratégias de gestão mais flexíveis e participativas e que incentivem e valorizem a atividade formal, e o desenvolvimento de uma estrutura que ofereça condições e ferramentas para que os profissionais e gestores desenvolvam novas competências para atender tais demandas.

Tais desafios são considerados multidimensionais e perpassam as dificuldades dos profissionais e das instituições, e afetam também as esferas política e econômica.

\section{Considerações finais}

Esta pesquisa, baseada em um scoping review, permitiu mapear as produções científicas brasileiras no que diz respeito à expressão 'gestão do trabalho em saúde', além de analisar o uso da expressão no contexto histórico.

Dos 34 artigos estudados, 31 foram desenvolvidos por autores vinculados a instituições de ensino e seus programas de pós-graduação. $\mathrm{O}$ número de publicações sobre o tema apresentou crescimento absoluto a partir de 2006, após a $3^{\mathrm{a}} \mathrm{CNGTES}$.

Por não existir o descritor 'gestão do trabalho em saúde', os estudos apresentaram grande variação de descritores e palavras-chave, o que pode ter conferido dificuldade na identificação de algum estudo relevante e, ainda, impedido a padronização na indexação de tais estudos. Recomenda-se, desta forma, a inclusão deste na lista BVS DeCS/MeSH.

Apesar de haver consenso a respeito da complexidade dos conceitos que envolvem o tema, foi possível notar que são explorados variados aspectos, de acordo com a interpretação de cada autor dependendo das mudanças políticas, sociais e econômicas que estavam ocorrendo no momento em que o pensamento foi construído. 
De maneira geral, os autores concordam que essa expressão aborda temas relacionados ao trabalhador e seu trabalho, incluindo sua valorização por meio da regulação e desprecarização das atividades profissionais, além de considerar toda sua vida funcional desde seu ingresso até a garantia dos direitos trabalhistas, perpassando capacitação, formação, participação nos processos de trabalho e nas formulações de políticas.

Sendo assim, a incorporação dessa expressão apresenta um sentido amplo, uma vez que compreende não somente o trabalho, mas quem o executa, como executa e sob quais condições, considerando todas as suas interfaces.

Em algumas publicações os termos 'gestão de recursos humanos', 'gestão de pessoas', 'gestão das relações de trabalho' e 'gestão do trabalho' são considerados sinônimos (Junqueira et al., 2009; Mendonça et al., 2010; Scalco, Lacerda e Calvo, 2010; Junqueira et al., 2010), por outro lado, outros autores deixam clara a diferença entre esses termos (Benito e Licheski, 2009; Campos, 2010; Pereira, 2004; Pierantoni et al., 2008; Moyses e Machado, 2010; Lacaz et al., 2010). Mello e Amâncio Filho (2010) afirmam que a política de recursos humanos está incluída entre as dimensões da gestão do trabalho, pois diz respeito aos seus aspectos administrativos.

A expressão 'recursos humanos', consagrada na literatura clássica sobre administração, recebe forte carga do modelo de gestão taylorista/fordista, em que, de forma funcional, os profissionais são considerados como um dos 'recursos' da cadeia produtiva, assim como os materiais e os financeiros, lógica' segundo a qual' a organização do trabalho tem, como característica, o predomínio da atividade prescrita, com pouca autonomia por parte de quem a executa.

De maneira geral, constatou-se o consenso entre praticamente todos os estudos incluídos nesta pesquisa de que, independente dos termos utilizados e de seus conceitos, a área que diz respeito aos trabalhadores e sua gestão deve receber atenção prioritária para que os problemas relativos à gestão dos serviços de saúde, principalmente no sistema público, sejam superados, e que as relações de trabalho sejam plenamente regulamentadas, considerando-se as peculiaridades específicas do setor com vistas a uma efetiva reforma do Estado brasileiro.

Por fim, destaca-se a necessidade de pensar quais são os reflexos dessa discussão teórica e conceitual na prática das organizações de saúde e se existe, de fato, diferença estratégica quando a gestão do trabalho é implementada em detrimento de outras formas de gestão (departamento de pessoal, gestão de recursos humanos ou gestão de pessoas, entre outras).

As discussões teóricas sobre o tema deveriam estimular mudanças nas práticas de gestão e na cultura herdada dos modelos burocráticos e de produção em massa, porém as aplicações práticas ainda são escassas, restando um questionamento que ainda não foi totalmente desvelado: gestão do trabalho em saúde é apenas uma mudança terminológica ou é uma mudança de paradigma? 


\section{Colaboradores}

Dirce Laplaca Viana participou de todas as etapas de planejamento, desenvolvimento, execução da pesquisa, elaboração e revisão do artigo. Cleide Lavieri Martins e Paulo Frazão orientaram o planejamento e a realização da pesquisa, bem como e revisão do artigo. Os autores declaram não haver conflito de interesse.

Resumen En Brasil, el término 'gestión del trabajo' pasó a ser utilizado después de la 12 $2^{\text {a }}$ Conferencia Nacional de Salud en sustitución a los 'recursos humanos'. El objetivo fue analizar, con base en el contexto histórico brasileño, los sentidos atribuidos a la expresión 'gestión del trabajo en salud' en la literatura científica brasileña. Esta encuesta ha desarrollado un scoping review en la base Lilacs, entre 1990 y 2010. De 436 estudios identificados, 34 fueron incluidos. Hubo crecimiento absoluto de las publicaciones después de la $3^{\text {a }}$ Conferencia Nacional de Gestión del Trabajo y Educación en Salud, 2006. El contenido fue sintetizado en cinco unidades temáticas: conceptos de gestión del trabajo en salud, formulación de políticas, participación de los trabajadores en los procesos de trabajo, precariedad del trabajo y nuevos desafíos. Se observó consenso sobre la complejidad de la expresión y se exploraran aspectos distintos dependiendo de las características políticas, sociales y económicas al momento de la construcción del pensamiento. Las discusiones teóricas deberían estimular cambios en las prácticas de gestión y en la cultura de los modelos de producción, sin embargo los cambios son escasos, restando un cuestionamiento no dado a conocer: gestión del trabajo en salud, un cambio terminológica o de paradigma?

Palabras clave administración de recursos humanos; administración de recursos humanos en salud; gestión del trabajo en salud; scoping review.

\section{Notas}

${ }^{1}$ Universidade de São Paulo, Faculdade de Saúde Pública, São Paulo, SP, Brasil.

$<$ dirce.laplaca@gmail.com>

Correspondência: Rua dos Jacintos, 497, Mirandópolis, CEP 04049-050, São Paulo, SP, Brasil.

${ }^{2}$ Universidade de São Paulo, Faculdade de Saúde Pública, Departamento de Prática de Saúde Pública, São Paulo, SP, Brasil.

<cleide@usp.br>

${ }^{3}$ Universidade de São Paulo, Faculdade de Saúde Pública, Departamento de Prática de Saúde Pública, São Paulo, SP, Brasil.

<pafrazao@usp.br>

${ }^{4}$ Artigo extraído da tese de doutorado intitulada Gestão do trabalho em saúde: revisão da literatura por meio de scoping review, de autoria da primeira autora deste artigo. Não houve necessidade de aprovação em Comitê de Ética em Pesquisa. 


\section{Referências}

ARKSEY, Hilary; O'MALLEY, Lisa. Scoping studies: towards a methodological framework. International Journal of Social Research Methodology, Oxford, v. 8, n. 1, p. 19-32, fev. 2005. Disponível em: <http:// eprints.whiterose.ac.uk/1618/1/Scopingstudies. pdf $>$. Acesso em: 4 abr. 2016.

BARBOSA, Nelson B. Regulação do trabalho no contexto das novas relações público versus privado na saúde. Ciência \& Saúde Coletiva, Rio de Janeiro, v. 15, n. 5, p. 2.497-2.506, ago. 2010.

BENITO, Gládys A. V.; LICHESKI, Ana P. Sistemas de informação apoiando a gestão do trabalho em saúde. Revista Brasileira de Enfermagem, Brasília, DF, v. 62, n. 3, p. 447450, maio -jun. 2009.

BRASIL. Ministério da Saúde. $8^{a}$ Conferência Nacional de Saúde: relatório final. Brasília, DF, 1986a. Disponível em: <http://conselho. saude.gov.br/biblioteca/Relatorios/relatorio_8. pdf> Acesso em: 19 dez. 2016.

BRASIL. Ministério da Saúde. Secretaria Geral. Secretaria de Recursos Humanos. Conferência Nacional de Recursos Humanos para a Saúde: relatório final. Brasília, DF, 1986b. Disponível em: <http://bvsms.saude.gov.br/bvs/ publicacoes/0116conf_rh.pdf $>$. Acesso em: 28 jul. 2012.

BRASIL. Ministério da Educação. Coordenação de Aperfeiçoamento de Pessoal de Nível Superior. Plano Nacional de Pós-Graduação: PNPG 2011-2020. v. 1. Brasília, DF: Capes, 2010. p. $45-80$.

BRASIL. Ministério da Saúde. Conselho Nacional de Saúde. $10^{a}$ Conferência Nacional de Saúde: relatório final, Brasília, DF, 1998. 91 p.

BRASIL. Ministério da Saúde. Conselho Nacional de Saúde. $12^{a}$ Conferência Nacional de Saúde: Conferência Sérgio Arouca. Brasília, 7 a 11 de dezembro de 2003: relatório final, Brasília, DF, 2004a. 232 p.
BRASIL. Ministério da Saúde. Secretaria de Gestão do Trabalho e da Educação na Saúde. Departamento de Gestão da Educação na Saúde. Política de educação e desenvolvimento para o SUS: caminhos para a educação permanente em saúde: polos de educação permanente em saúde. Brasília, DF, 2004b. 68p. (Série C. Projetos, Programas e Relatórios)

BRASIL. Ministério da Saúde. Conselho Nacional de Saúde. Princípios e diretrizes para a gestão do trabalho no SUS (NOB/RH - SUS). 3. ed. Brasília, DF, 2005. 98 p.

BRASIL. Ministério da Saúde. Secretaria de Gestão do Trabalho e da Educação na Saúde. Departamento de Gestão e da Regulação do Trabalho em Saúde. Programa Nacional de Desprecarização do Trabalho no SUS: DesprecarizaSUS - perguntas \& respostas. Comitê Nacional Interinstitucional de Desprecarização do Trabalho no SUS. Brasília: Editora do Ministério da Saúde, 2006. 28p.

CAMPOS, Gastão W. de S. Cogestão e neoartesanato: elementos conceituais para repensar o trabalho em saúde combinando responsabilidade e autonomia. Ciência \& Saúde Coletiva, Rio de Janeiro, v. 15, n. 5, p. 2.337-2.344, ago. 2010.

CAMPOS, Gastão W. de S.; DOMITTI, Ana Carla. Apoio matricial e equipe de referência: uma metodologia para gestão do trabalho interdisciplinar em saúde. Cadernos de Saúde Pública, Rio de Janeiro, v. 23, n. 2, p. 399407, fev. 2007.

DOMINGUEZ, Bruno C. A desprecarização é urgente. Radis: Comunicação em Saúde, Rio de Janeiro, n. 46, p. 8-11, jun. 2006.

DUARTE, Ivomar G.; BOTAZZO, Carlos. Gestão de pessoas nas Organizações Sociais de Saúde: algumas observações. Revista de Administração em Saúde, São Paulo, v. 11, n. 45, p. 161-168, out./dez. 2009. 
FERNANDES, Marcelo C. et al. Análise da atuação do enfermeiro na gerência de unidades básicas de saúde. Revista Brasileira de Enfermagem, Brasília, DF, v. 63, n. 1, p. 11-15, jan./fev. 2010

FISCHER, Rosa M. Gestão do trabalho: dimensões institucionais e organizacionais. Revista de Administração de Empresas, São Paulo, v. 31, n. 4, p. 85-90, out.-dez. 1991.

FUJITA, Mariângela S. L. et al. (org.). $A$ indexação de livros: a percepção de catalogadores e usuários de bibliotecas universitárias. Um estudo de observação do contexto sociocognitivo com protocolos verbais. São Paulo: Editora Unesp, 2009. 149 p.

GARIBOTTI, Vanda; HENNINGTON, Élida A.; SELLI, Lucinda. A contribuição dos trabalhadores na consolidação dos serviços municipais de vigilância sanitária. Cadernos de Saúde Pública, Rio de Janeiro, v. 22, n. 5, p. 1043-1051, maio 2006.

HENNINGTON, Élida A. Gestão dos processos de trabalho e humanização em saúde: reflexões a partir da ergologia. Revista de Saúde Pública, São Paulo, v. 42, n. 3, p. 555-561, jun. 2008.

JASMIN, Marcelo G. História dos conceitos e teoria política e social: referências preliminares. Revista Brasileira de Ciências Sociais, São Paulo, v. 20, n. 57, p. 27-38, fev. 2005.

JORGE, Maria S. B. et al. Gestão de recursos humanos nos centros de atenção psicossocial no contexto da política de desprecarização do trabalho no Sistema Único de Saúde. Texto \& Contexto Enfermagem, Florianópolis, v. 16, n. 3, p. 417-425, jul.-set. 2007.

JUNQUEIRA, Túlio da S. et al. As relações laborais no âmbito da municipalização da gestão em saúde e os dilemas de relação expansão/ precarização do trabalho no contexto do SUS. Cadernos de Saúde Pública, Rio de Janeiro, v. 26, n. 5, p. 918-928, maio 2010.

JUNQUEIRA, Túlio da S. et al. Saúde, democracia e organização do trabalho no contexto do Programa de Saúde da Família: desafios estratégicos. Revista Brasileira de Educação Médica, Rio de Janeiro, v. 33, n. 1, p. 122-133, jan.-mar. 2009.

KOSELLECK, Reinhart. Uma história dos conceitos: problemas teóricos e práticos. Estudos Históricos. Rio de Janeiro, v. 5, n. 10, p. 134146, jul.-dez. 1992.

LACAZ, Francisco A. de C. et al. Qualidade de vida, gestão do trabalho e plano de carreira como tecnologista em saúde na atenção básica do Sistema Único de Saúde em São Paulo, Brasil. Cadernos de Saúde Pública, Rio de Janeiro, v. 22, n. 2, p. 253-263, fev. 2010.

MACHADO, Maria H., Gestão do trabalho em saúde no contexto de mudanças. Revista de Administração Pública, Rio de Janeiro, v. 34, n. 4, p. 133-146, jul.-ago. 2000.

MACHADO, Maria H., KOSTER, Isabela. Emprego e trabalho em saúde no Brasil: as políticas de desprecarização do Sistema Único de Saúde. In: ASSUNÇÃO, Ada A.; BRITO, Jussara. Trabalhar na saúde: experiências cotidianas e desafios para a gestão do trabalho e do emprego. Rio de Janeiro: Editora Fiocruz, 2011. p. 195-215.

MACHADO, Maria H., PAULA, Aïda E.; AGUIAR FILHO, Wilson. O trabalho em saúde no Mercosul: uma abordagem brasileira sobre a questão. Cadernos de Saúde Pública, Rio de Janeiro, v. 23, supl. 2, p. S292-S301, 2007.

MATOS Eliane; PIRES, Denise. Teorias administrativas e organização do trabalho: de Taylor aos dias atuais, influências no setor saúde e na enfermagem. Texto \& Contexto Enfermagem, Florianópolis, v. 15, n. 3, p. 508-514, jul.-set. 2006.

MEDEIROS, Adriane C. et al. Gestão participativa na educação permanente em saúde: olhar das enfermeiras. Revista Brasileira de Enfermagem. Brasília, DF, v. 63, n. 1, p. 38-42, jan.-fev. 2010. 
MELLO, Márcio L. B. C.; AMÂNCIO FILHO, Antenor. A gestão de recursos humanos em uma instituição pública brasileira de ciência e tecnologia em saúde: o caso Fiocruz. Revista de Administração Pública, Rio de Janeiro, v. 44, n. 3, p. 613-636, maio-jun. 2010.

MENDONÇA, Maria H. M. et al. Desafios para a gestão do trabalho a partir de experiências exitosas de expansão da Estratégia de Saúde da Família. Ciência \& Saúde Coletiva, Rio de Janeiro, v. 15, n. 5, p. 2.355-2.366, ago. 2010.

MOYSES, Neuza M. N.; MACHADO, Maria Helena. Políticas de gestão do trabalho no SUS: o desafio sempre presente. Divulgação em Saúde para Debate, Londrina, v. 45, p. 33-41, maio 2010.

NOGUEIRA, Arnaldo J. F. M. Gestão estratégica das relações de trabalho. In: FLEURY, Maria T. L. (coord.). As pessoas na organização. São Paulo: Gente, 2002. p. 102-137.

PEREIRA, Larissa D. A gestão da força de trabalho em Physis: Revista de Saúde Coletiva, Rio de Janeiro, v. 14, n. 2, p. 363-382, jul.-dez. 2004. Saúde na década de 90.

PIERANTONI, Célia R. As reformas do Estado, da saúde e recursos humanos: limites e possibilidades. Ciência \& Saúde Coletiva, Rio de Janeiro, v. 6, n. 2, p. 341-360, jul./ dez. 2001.

PIERANTONI, Célia R. et al. Gestão do trabalho e da educação em saúde: recursos humanos em duas décadas do SUS. Physis: Revista de Saúde Coletiva, Rio de Janeiro, v. 18, n. 4, p. 685-704, out.-dez. 2008.

PIMENTA, Aparecida L.; LIVORATO, Fátima. Educação permanente: ferramenta para qualificar os sistemas municipais de saúde. Divulgação em Saúde para Debate, Londrina, v. 44, p. 85-99, maio 2009.

PIRES, Denise. Reestruturação produtiva e consequências para o trabalho em saúde. Revista Brasileira de Enfermagem, Brasília, DF, v. 53, n. 2, p. 251-263, abr.-jun. 2000.
PIRES-ALVES Fernando; PAIVA, Carlos H. A.; HOCHMAN, Gilberto. História, saúde e seus trabalhadores: da agenda internacional às políticas brasileiras. Ciência \& Saúde Coletiva, Rio de Janeiro, v. 13, n. 3, p. 819-829, maio-jun. 2008.

RAMOS, Alexandre de S. et al. Política de gestão do trabalho e educação permanente na Bahia: "o SUS é uma escola". Revista Baiana de Saúde Pública, Salvador, v. 33, n. 1, p. 40-50, jan.-mar. 2009.

RUBI, Milena P. Política de indexação para construção de catálogos coletivos em bibliotecas universitárias. 2008. 169 f. Tese (Doutorado em Ciência da Informação) - Faculdade de Filosofia e Ciências, Universidade Estadual Paulista, Marília, 2008.

SANTANA, José P. Gestão do trabalho no serviço público de saúde. Saúde em Debate, Rio de Janeiro, n. 37, p. 66-69, dez. 1992.

SARAIVA Enrique. Introdução à teoria da política pública. In: SARAIVA Enrique; FERRAREZI Elisabete (org.). Políticas públicas. Brasília: Escola Nacional de Administração Pública, 2006. v. 1, p. 21-42.

SCALCO, Sirlesia V.; LACERDA, Josimari T.; CALVO, Maria C. M. Modelo para avaliação da gestão de recursos humanos em saúde. Cadernos de Saúde Pública, Rio de Janeiro, v. 26, n. 3, p. 603-614, mar. 2010.

SCHERER, Magda D. A.; PIRES, Denise; SCHWARTZ, Yves. Trabalho coletivo: um desafio para a gestão em saúde. Revista de Saúde Pública, São Paulo, v. 43, n. 4 , p. 721-725, ago. 2009.

SILVEIRA, Denise S. et al. Gestão do trabalho, da educação, da informação e comunicação na atenção básica à saúde de municípios das regiões Sul e Nordeste do Brasil. Cadernos de Saúde Pública, Rio de Janeiro, v. 26, n. 9 , p. 1.714-1.726, set. 2010.

SODRÉ, Francis. Serviço social e o campo da saúde: para além de plantões e encaminhamentos. Serviço Social \& Sociedade, São Paulo, n. 103 , p. 453-475, jul.-set. 2010. 
TIMBÓ, Dina B. P. et al. As competências para o gerenciamento eficaz: o caso da Rede de Serviços de Saúde São Gerardo. Revista de Administração em Saúde, São Paulo, v. 11, n. 43, p. 72-82, abr.-jun. 2009.

VIANA, Dirce L. Gestão do trabalho em saúde: revisão da literatura por meio de scoping review. 2013. 170 f. Tese (Doutorado em Saúde Pública) - Faculdade de Saúde Pública da Universidade de São Paulo, São Paulo, 2013.
VIEGAS, Selma M. F. et al. O cotidiano da assistência ao cidadão na rede de saúde de Belo Horizonte. Physis Revista de Saúde Coletiva. Rio de Janeiro, v. 20, n. 2, p. 769-784, abr./ jun. 2010.

VIEIRA, Monica. Trabalho, qualificação e a construção social de identidades profissionais nas organizações públicas de saúde. Trabalho, Educação e Saúde, Rio de Janeiro, v. 5, n. 2, p. 243-260, jul. 2007.

Recebido em 08/04/2016. Aprovado em 08/04/2017. 